\title{
SOBRE LA MODALIDAD AUTOBIOGRÁFICA EN SARMIENTO
}

La condición de existencia de la autobiografía como género literario exige la igualación de autor-narrador-personaje, y postula su identidad. Sin embargo, la disyunción entre tiempos, espacios y situaciones de la narración y de lo narrado niega la posibilidad de que esa igualdad se dé. Esto se complica con otra sutil disyunción entre el nivel de la narración y el de la escritura, surgida de la especial empresa del autor que se elige a sí mismo como doble objeto: como protagonista de la historia contada y como escritor que produce un texto, focalizando dos escenas, la escena del actuar y la escena del escribir. Tal es la paradoja constitutiva del género autobiográfico: la tensión entre la identidad de sus tres elementos (nacida de una empresa del hombre a la busca de su unidad central, la historia de su yo) y la dispersión, la nueva tensión de su doble rôle (acción-escritura), el desgaste, la distorsión bajo las presiones de otras fuerzas ajenas, el fluir temporal aleatorio. En ello reside su complejidad y su riqueza ${ }^{1}$.

I Para un intento de precisión del género autobiográfico puede consultarse Philippe LeJeune, Le pacte autobiographique, Paris, Seuil, 1975 y su abundante bibliografía, junto con la de Autobiography. Essays theoretical and critical, editados por James Olney, Princeton, 1980, en donde se recomienda también consultar la nueva variante que expone Elizabeth W. Bruss, de las reglas que antes propuso para definir la autobiografía según tres parámetros: "Truth-value: An autobiography purports to be consistent with other evidence; we are conventionally invited to compare it with other documents that describe the same events (to determine its veracity) and with anything the author may have said or written on other occasions (to determine its sincerity). Act-value: Autobiography is a personal performance, an action that exemplifies the character of the agent responsible for that action and how it is performed. Identity-value: In autobiography, the logically distinct roles of author, narrator, and protagonist are conjoined, with the same individual occupying a position both in the context, the associated «scene of writing», and within the text itself" (pp. 299-300). Para el desdoblamiento del sujeto de la narración y el sujeto de lo narrado, véase el artículo clásico de JEAN STARo. ninski, "Le style de l'autobiographie", Poétique, 3 (1970), 257-265. 
Todos los críticos están de acuerdo en el carácter marcadamente autobiográfico de la obra de Sarmiento. Sin embargo queda bastante por hacer para precisar la originalidad que distingue las modalidades autobiográficas de su obra.

Un rasgo de ellas que quiero destacar es la explícita relación vida-escritura. Este rasgo, que ha llegado a ser común y a veces marcadamente retórico en la literatura contemporánea, no era usual en la tradición literaria hispánica del siglo xIX. Bastará el comentario del "Prólogo" a la Campaña en el Ejército Grande para mostrar el intrincado juego de referencias que se establece, y los planos en que se mueve el narrador-autor-personaje.

Un breve prolegómeno (prólogo al prólogo) generaliza la actitud de desconcierto de los que pasan de la inconsciencia del sueño letárgico o el desvanecimiento del herido grave, a la confrontación con la realidad. Sus líneas se mueven ya, con una estrategia particular de Sarmiento, entre la anécdota risueña (aquí "el puf norteamericano") y la amarga alusión a las crueldades de las luchas civiles.

El exordio de experiencia generalizadora sólo ha servido para fijar al autor y al lector en el momento de la iniciación de la escritura.

Sucédenle cosas a uno en la política americana que no sería extraño tomarse despierto, bien despierto, por el negro del cuento, experimentando realmente aquella desorientación de que hablaba al principio; y vale la pena de contarlo, la fascinación, que, después de disipada, me ha inducido a poner orden por escrito a mis últimas reminiscencias (p. 54).

Se ha fijado el momento en el vacío, se diría, dejando como una incógnita sin aclarar, las circunstancias de la experiencia.

Después de esto, la vuelta al pasado reintroduce el caso particular "vivido" por el narrador-autor, la quiebra de la continuidad de la conciencia y el laborioso proceso de recuperarla en un viaje de reconstrucción hacia ese pasado. Entendámonos: las comillas de "vivido" nos alertan contra la trampa de interpretar estas

2 Cito (con indicación del número de página entre paréntesis) Campaña en el Ejército Grande Aliado de Sud América, por la edición con prólogo y notas de Tulio Halperín Donghi, México-Buenos Aires, 1958, que sigue más fielmente las publicaciones originales de 1852 (en sus tres entregas sucesivas) que la edición nacional, Obras de D. F. Sarmiento publicadas bajo los auspit cios del Gobierno Argentino, t. 14, Buenos Aires, Imprenta y Litografía Mariano Moreno, 1897, al cuidado de A. Belín Sarmiento. El prólogo de Halperín es la mejor sintesis evaluativa existente de la obra sarmientina. (Los subrayados son míos.) 
curiosas páginas como un caso de buceo en el análisis psicológico o en los minuciosos mecanismos de la memoria reconstructiva. Son sí, como se verá, una muestra perfecta de las estrategias del narrador-autor en sus relaciones con el narratario-lector para ofrecerle y hacerle aceptar la imagen personal que quiere construir.

Así continúa: "Un sueño pesado me había retenido uno de esos días en el lecho, hasta muy avanzada la mañana. Hube de abrir al fin los ojos con dificultad, y a mi frente y sirviéndole de marco el claro de una ventana, presentóseme un cuadro natural y para mí desconocido" (p. 55). Y tras un largo párrafo en que describe un paisaje exuberante y cálido pero al mismo tiempo cuidado y embellecido por la mano del hombre, comparable a los paisajes de la India, Madrás o Calcuta, "donde la cultura inglesa ha sometido a regla la naturaleza tropical" (p. 55), se pregunta dónde está, sin haber podido disipar el letargo, y se reconoce despierto y al mismo tiempo totalmente confuso.

El trabajo de recuperación de la conciencia, le hace dar por otra quiebra brusca, un nuevo salto a un pasado más alejado aún:

No pudiendo tomar por el próximo extremo el hilo interrumpido de mi existencia, empecé a buscarlo un poco más allá, entre mis recuerdos, y pude al fin cerciorarme de que no hacía aún seis meses, éramos siete que partimos de Chile, rondando el Cabo de Hornos, a bordo de la Médicis, a prestar nuestros servicios al general Urquiza contra el tirano argentino (p. 56).

El salto de seis meses sólo tiene por objeto introducir la prehistoria de la Campaña en el Ejército Grande, que es al mismo tiempo la pre-historia y parte de la historia de la guerra para derrotar a Rosas (los hechos vividos) y la pre-historia del texto hasta el momento de su escritura.

En efecto, el narrador-autor introduce en este punto dos microautobiografías, o si se quiere una micro-autobiografía repetida en dos claves. Digo en dos claves porque la primera está redactada en tercera persona, característica que suele encontrarse en Sarmiento cuando toma distancia con respecto a sí mismo y se considera como un personaje histórico o como una figura arquetípica del hombre público. Así se incluye englobado en el grupo de los emigrados de Chile, embarcados en la Médicis en Valparaíso para acudir a la guerra que culminó en Caseros ${ }^{3}$. "El capitán retirado de

3 A Sarmiento ie interesa ponerse a la par de militares de carrera superiores a él, reconocidos por sus campañas guerreras y también, algunos, por su importancia política: "Aquino, el brillante y caballeresco coronel [...]; el coronel Paunero, experimentado soldado de la guerra del Brasil; el teniente 
Coraceros de la Guardia, Domingo F. Sarmiento" es uno de los militares distinguidos a la par de Paunero y Mitre, a la par de los que afrontaron el peligro hasta la muerte ("De estos siete soldados han muerto dos") y de ellos (de él incluido entre ellos), se resume en breves renglones un periplo glorioso, casi épico, con sus notas de hidalgo desinterés y gallardía.

Los que han sobrevivido halláronse a la sombra del pabellón imperial en el combate naval del Tonelero, y arrostrando las balas rojas, la fusilería y metralla de Mansilla, durante cincuenta y cinco minutos, y en la batalla campal de Monte Caseros, a las órdenes del victorioso general Urquiza, hecho cuanto puede esperarse de hombres de pio y de soldados de honor; viéndoseles entre los jefes, y haciendo la campaña a sus propias expensas con sus armas y caballos, como los antiguos capitanes castellanos (p. 56) .

En la segunda micro-autobiografía, redactada en primera persona, el narrador-autor-personaje, recobrada su unidad, retoma su caso, lo aísla del grupo, lo particulariza, y despliega nuevamente su periplo en una página que realiza en escasas líneas el milagro de un discurso sintético y de un gesto vital amplio y cargado de experiencias, que remata en el preciso momento de la escritura. Imposible dejar de transcribir el párrafo completo, porque sólo su lectura puede dar idea del juego de elementos movilizados.

Por lo que a mí respecta, pues ya sabía quién yo era, traje a la memoria, al volver de mi trascuerdo, que, dejando atrás familia y cuidados de fortuna, en busca de una patria libre y crita, por quince años de destierro suspirada, había costeado el Atlántico y el Pacífico, remontado el majestuoso Uruguay y el fecundizante $\mathrm{Pa}$ raná; atravesado las provincias argentinas Entre Ríos y Santa Fe; visitado las capitales Montevideo y Buenos Aires; batídome en mar y en tierra; y, viajando y combatiendo, soportado duras fatigas, y gozado de emociones profundas; observando lo que mis ojos veian y oian mis oidos; pensando, escribiendo, y viviendo de la vida febril del entusiasmo y de la lucha; y como si algo faltara en este vivísimo panorama, pasado a mi vista en cinco meses de actividad y movimiento, a los hielos del Cabo de Hornos, venían por añadidura a oponerse los esplendores sofocantes del trópico, y a las desnudas e ilimitadas planicies de las pampas argentinas las sañudas crestas y picos, que, entre bosques enmarañados, rodean la lujosa bahía de Río de Janeiro, donde escribo estas páginas, en el Catete,

coronel Mitre, maestro profundo en su arma, la artillería" (p. 56). Más adelante Aquino volverá a primer plano, por su romántico, funesto y fantasmal destino. Para un fino análisis de la autobiografía en tercera persona véase Philippe Lejeune, "L'autobiographie à la troisième personne", en je est un autre, Paris, 1980, pp. 32-59. 
barrio pintoresco y fashionable, Hotel des Etrangers, en una habitación alegre cuyas ventanas dan hacia el pedazo de mar, contenido entre los faldeos de la montaña Das Orgas, el Pan de Azúcar y el Corcovado, y era la taza de agua que en parte caía bajo mis miradas al despertar, $y$ no acertiba a comprender en el primer momento (pp. 56-57).

Hemos vuelto como se ve al punto en que empieza a engendrarse el texto de la Campaña en el Ejército Grande. De los elementos desplegados en este párrafo, no es el menos importante la vasta geografía recorrida por el "peregrino", los contrastes y la variedad de climas y de paisajes (ríos, montañas, llanuras con sus dimensiones colosales). Están también los afectos y las conveniencias materiales sacrificadas, la angustia del largo destierro, las emociones y las hazañas guerreras. Parte de las líneas subrayadas por mi (las primeras) quieren destacar lo que Sarmiento quiso siempre destacar y destacó en todas sus manifestaciones autobiográficas como una constante reveladora de su personalidad: que su capacidad de acción concreta estaba sustentada siempre por su capacidad de observar, meditar sobre lo observado y volcar en un discurso coherente sus conclusiones como un programa para la acción: observar-pensar-escribir-luchar.

Pero volver al punto en que se engendra el texto, como lo indican las otras líneas finales del párrafo que también subrayét, es volver a insertarse en la categoría de "desterrado" ("emigrado", "prófugo", "proscripto", "peregrino"), junto con la de escritor.

No comentaré todo el prólogo (las fatídicas premoniciones del asesinato de Aquino y sus apariciones fantasmales more romantico), el tema obsesivo de la divisa colorada y del orden rosista como un sueño o como una peste contagiosa, ni aun la exaltación de su lucha por la prensa, igualada en eficacia a la acción militar contra el tirano.

Quiero detenerme en otro párrafo significativo de la intrincada relación de los sujetos de la enunciación y de lo enunciado entre sí y con el texto, junto con la intensificación del proceso del discurso. Durante los vaivenes de la campaña, al acampar el ejército cerca de Puente Márquez, llegó a manos de Rosas el cuaderno en que Sarmiento consignaba sus impresiones diarias en forma telegráfica, al caer prisionero el asistente que llevaba sus papeles, y sólo lo recuperó después de Caseros $^{\mathbf{5}}$. A este incidente se refiere

4 En efecto, en la p. 55 habia hablado de "una inmensa taza de agua tersa [...] agitada en partes por barquillas de dos velas latinas", que veía desde la ventana al despertar, sin poder saber dónde estaba, ni reconstruir el derrotero anterior de su vida.

5 Así en la introducción a sus Obras, t. 14, p. 9, de A. Belín Sarmiento. 
cuando ha llegado en el final del "Prólogo" el momento de presentar el libro concluso a los lectores.

Llama la atención nuevamente la doble o triple actitud de quien resume (y esculpe) para la posteridad su vida (su estatua), $y$ al hacerlo une en forma indisoluble (porque una cosa implica la otra) el batallar y el escribir, la pasión ("fulminar") y el razonamiento ("las causas"), con lo que paradójicamente se distancia de su experiencia y se sumerge en ella al mismo tiempo.

Soldado, con la pluma o la espada, combato para escribir, que escribir es pensar; escribo como medio y arma de combate, que combatir es realizar el pensamiento, y este mi titulado Diario de la Campaña en el Ejército Grande tiene por objeto dar cuenta a mis amigos de los hechos a que se refiere como de las causas que los produjeron, y los resultados que debiera dar y dará el triunfo de Monte Caseros, a que concurrí con mi doble carácter, arrastrando desde el Pacífico al campo de batalla aquella prensa de Chile que continuó fulminando y persiguiendo al tirano hasta las calles de Buenos Aires (pp. 61-62).

En este punto, el libro que se ofrece al lector argentino (bajo la máscara de "a mis amigos") es un bloque estático y concluso. Pero no se le deja descansar en tal estatismo: por un nuevo giro asistimos a otro avatar más curioso y original.

Tienen estos apuntes la gloria y la recomendación de haber pasado en resumen por la vista de don Juan Manuel de Rosas, la víspera de la batalla, como si hubiese sido la mala suerte de aquel pobre hombre que yo había de estarle zumbando al oído: jcaerás... ya caes... ya has caído! pues lo que leía en manuscrito estaba destinado para ver la luz después de su caída (p. 62).

El texto pone en escena al personaje encmigo en el acto de leer su propia historia, pero no como un cuadro fijo sino transformándolo en un proceso en que el narrador-escritor se trasmuta

Paul Vérdevoye, en "Diario de la Campaña del Tte. Cnel. D. F. Sarmiento en el Ejército Grande de Sud América (1852)", en Les cultures ibériques en devenir. Essais publiés en hommage à la mémoirc de Marcel Bataillon par la Fondation Singer Polignac, Paris, 1979, pp. 603-615, lo publicó íntegro e in dicó que lleva una nota autógrafa en la contratapa: "Cayó en poder / de Rosas el 1ro. de $\mathrm{Feb}^{\circ}$ de 1852. / Rescatélo el 3 después de la batalla de MonteCaseros / Sarmiento", p. 603. Existe una publicación anterior parcial en el folleto de Antonio P. Castro, Sarmiento y Urquiza, Buenos Aires, Museo His. tórico Sarmiento, 1954, serie II, núm. 25, donde Castro anota que lo había perdido junto con la carta topográfica el 30 de enero, en p. 21. Ias interpretaciones acerca de las relaciones de Sarmiento y Urquiza difieren en Castro, Halperín y Verdevoye. 
en un personaje-narwador, el personaje odiado en un personaje-narratario, el texto en un discurso oralizado en devenir, que liga a los enemigos y se desenvuelve ante los ojos de los lectores en su trascurrir temporal, acelerado y amenazante. Hoy es tan común para el lector la experiencia de la autorreferencialidad de los textos, que a veces no percibimos la originalidad de la escritura de Sarmiento en su lugar y en su época.

Otro último golpe de timón trasmuta el libro en texto histórico, aprobado (según da por supuesto) por uno de los protagonistas de los sucesos reales que lo avala (¿avala su verdad y acaso también su valor literario?: "bueno") y al mismo tiempo lo convierte en un objeto mágico y en un objeto simbólico de la historia argentina que revierte siniestramente sobre la fuente del discurso.

[Rosas] Debió hallarlo, sin embargo, bueno y verídico, pues no lo rompió, y pude rescatarlo entre los despojos del combate, y hallar todos mis papeles, según la minuta del general Pacheco, en orden; y icosa extraña y fatídica! jamarrados todos con una ancha cinta colorada! ¿Mandábame Rosas en ella el cordón morado que debía amargar nuestro triunfo?

Ello es que, a causa de su fatal don, tuve que seguirle a poco; como él, asilarme en un buque de guerra; como él contemplar tristemente a Buenos Aires tres días desde las balizas; como él, decir adiós a la patria y tomar el camino del extranjero... (p. 62).

El libro como objeto revierte sobre su narrador-autor-personaje, para destacar el último de su triple papel, y poder así volver a ligarlo con el personaje enemigo como condenados infernales, igualados verdugo y víctima, atados al mismo yugo en un mismo destino peregrinante de proscritos, ahora que se levanta un nuevo enemigo con el poder ascendente de Urquiza, el caudillo entrerriano.

Dije antes que observar-pensar-escribir-luchar, todo se funde en Sarmiento en un gesto que engloba la construcción de la propia vida y la construcción del país en que le tocó nacer, donde escribir la vida del uno es escribir la historia del otro, pero más sin duda, donde escribir se identifica con actuar: "Yo escribo la historia" dice en frase rotunda y a la vez de doble sentido en carta a Mrs. Mann, del 20 de mayo de 1866, a propósito de su Vida del Chacho, queriendo significar juntamente que él concluye con el caudillismo y que él escribe la "verdadera" interpretación histórica de los acontecimientos en que le tocó ser actor privilegiado ${ }^{\theta}$.

- Cartas de Sarmiento a la señora María Mann, Publicación de la Academia Argentina de Letras, Buenos Aires, Imprenta de la Universidad, 1936, p. 145. 
En 1843 Sarmiento publica $M i$ defensa, el primer texto que tiene el carácter de autobiografía bien definido, para responder a los ataques de don Domingo Godoy. En esa fecha es apenas el emigrado redactor de artículos periodísticos en El Mercurio, El Nacional y El Progreso y de algún folleto sobre métodos de lectura, escritor y educador incipiente, director de la Escuela Normal de Santiago de Chile?. Sin embargo ya se ve a sí mismo como hombre público, ya está pensando la construcción de su vida ligada a la construcción de su patria ${ }^{8}$. Ya puede quejarse amargamente de la lucha incesante en que la ruina del país -plano histórico- destruye lo que el propio esfuerzo había creado - plano personal:

7 Si se exceptúan los artículos publicados en El Zonda, en San Juan, en 1839, que se habían olvidado en Chile, sólo queda lo aparecido en los periódicos citados. Para la lista de folletos, libros, artículos y cartas, véase la bibliografía de 1839 a 1852, publicada por Paul Verdevoye en Domingo Faustino Sarmiento. Educateur et publiciste (entre 1832 et 1852), Paris, Institut des Hautes Études de l'Amérique Latine, 1963, pp. 498-541. Para Mi defensa y Recuerdos de provincia cito por Obras, t. 3, Santiago de Chile, Imprenta Gutenberg, 1885, con indicación de número de página entre paréntesis y la ortografía modernizada.

8 En carta dirigida a su nieto, Augusto Belín Sarmiento, el 14 de enero de 1873, define el voluntarismo que se fija como ideal moral de la conducta humana en su doble función privada y pública: "[...] y tomases la vida como una obra que un joven tiene entre manos, a fin de construir su porvenir y además llenar los deberes que la sociedad le trae impuestos", en JuLia OтtoL.ENGHI, Sarmiento a través de un epistolario, Buenos Aires, 1939, p. 105. Luis Franco, Sarmiento entre dos fuegos, Buenos Aires, 1968, p. 322, recuerda una cita de Obras, t. 42, que no he podido localizar: "A un avaro se le convertía en oro todo lo que tocaba, a mí se me vuelven hechos públicos hasta los más simples actos de la vida privada". Pedro Henríguez UReÑa, Las corrientes literarias en la América Hispánica, trad. de J. Diez Canedo, Buenos Aires, 1954, destacó esta característica de nuestra cultura hispanoamericana desde la independencia hasta fines del siglo xIx, con el advenimiento del modernismo, que coincide con el desarrollo económico y trae la división del trabajo: "Los intelectuales más típicos de este período fueron aquéllos a quienes podríamos llamar luchadores y constructores [..,] hombres que sabían ver en la literatura una parte de su servicio público" (p. 155). Véase también cap. 5: pp. 118, 120; cap. 6: pp. 148-149, 155-154; cap. 7: pp. 165, 166, 176-177. 191. Para una crítica ideológica del proceso, pueden consultarse la serie de obras de DAvio Viñas que llevan el título general de Literatura argentina y realidad politica, De Sarmiento a Cortázar, Buenos Aires, 1970, La crisis de la ciudad liberal, 1973, El apogeo de la oligarquia, 1975. Para el fenómeno general europeo del surgimiento después de la revolución francesa y con el romanticismo, de una corporación de los intelectuales como sustitutos laicos de la Iglesia en el papel de guías espirituales de la sociedad, véase Paur BÉnICHou, Le sacre de l'écrivain: 1750-1830, Paris, 1973, 
...la corta carrera que he podido andar, me la he abierto a fuerza de constancia, de valor, de estudios y de sufrimientos. [Ah! La mitad del tiempo lo he perdido en estos trabajos, tan improductivos como inevitables. [Cuando he logrado surgir para mi patria, ella se hunde bajo mis pies, se me evapora, se me convierte en un espectro horrible! (p. 5).

La busca de la reputación personal se presenta justificada no por sf misma, sino por la obra de bien público que se desea realizar:

Y si aún merezco tener una reputación, la necesito como una fortuna para mi propio bienestar, y, enseguida ofrecerla a la sociedad, para cimentar y difundir la educación a que he dedicado mis esfuerzos (p. 6).

Su imagen de la indisoluble unidad de la vida personal y la vida del pais encuentra más tarde un eco en Recuerdos de provincia (1850), en la sección dedicada a la evocación de los antepasados. Sus predecesores desfilan uno a uno convocados para reflejar en variados espejos biográficos una sola autobiograffa, la de la fuente del discurso que se oculta tras ellos.

El obispo Oro ha muerto, pues, prematuramente a los 65 años, habiendo gastado toda su vida en el penoso ascenso que de humilde fraile de un convento lo llevaba al obispado; mala estrella común a muchos hombres de mérito que tienen que levantar uno a uno todos los andamios de su gloria, crearse el teatro, formar los espectadores, para poder exhibirse enseguida. ¡Cuántas veces es destruida la obra, que es fuerza volver a comenzar! ¡Cuántos dfas y años pasados en presencia de un obstáculo que embaraza el pasol (pp. 7172).

Como habla pensado concluir $M i$ defens $a^{\ominus}$, Sarmiento concluye

9 Al fin de Mi defensa, en la 3ra. parte "El hijo, el hermano, y el amigo" Sarmiento anunciaba: "Ya he mostrado al hombre, tal como es, o como él mismo se imagina que es. En una segunda publicación mostraré al libelista famoso, al escritor en Chile, al maestro de escuela, mis obras últimamente, mis principios polfticos y sociales" (p. 23). Guillermo Ara, en su edición de Recuerdos de provincia, Buenos Aires, 1953, p. 288, interpreta que cumplió este propósito con el opúsculo "El escritor en Chile" y lo agrega junto con "Vaya un fresco (sic). Para don Domingo Godoy que ha caminado tanto estos días" y "El libelo", como apéndices para completar Mi defensa. Para ellos utiliza, según dice, la edición de las Obras completas, Buenos Aires, 1913, t. 3. Paul Verdevoye, en la bibliografía ya citada, sólo anota la existencia del folleto "Vaya un fresco. Progreso, Santiago, 2 pages"; aparte de "Mi defensa: Introducción, Mi infancia, El militar y el hombre de partido, 
Recuerdos de provincia con un balance de su obra como escritor, el rasgo que aún sigue predominando en la imagen que construye de sí mismo en esta época. Pero sin duda ya cuenta para entonces con un caudal importante de publicaciones cuya exposición interesa.

El espíritu de los escritos de un autor, cuando tiene un carácter marcado, es su alma, su esencia. El individuo se eclipsa ante esta manifestación, y el público menos interés tiene en los actos privados que en la influencia que aquellos escritos han podido ejercer sobre los otros (p. 204).

Entiéndase: el escritor importa, sin duda, pero se justifica sobre todo por la acción que puede ejercer a través de la palabra, modificando a los hombres, y también a la realidad toda, cuando le den la ocasión de transformarla. Después de la lista comentada en sus obras ("Diarios y publicaciones periódicas", "Folletos", "Biografías", "Libros", "Traducciones", "Casas de educación"), una micro-autobiografía cierra sus Recuerdos de provincia. Sólo copiaré un frag. mento significativo:

Tal es el cuadro modesto de mis pequeños esfuerzos en favor de la libertad y del progreso de la América del Sur, y como auxiliares poderosos, la educación de todos y la inmigración europea. Esfuerzos, es preciso decirlo, hechos a la par que luchaba con las dificultades de la vida para vivir, que combatía a los instrumentos de Rosas para tener patria, que educaba mi espíritu para com. pletar mis ideas; esfuerzos que en la América del Sur no son co. munes ni por la constancia y tenacidad de ellos, ni por la homogeneidad; esfuerzos que desde el primer día hasta el último, desde el primer artículo de un diario, hasta la última página de un libro, forman un todo completo; variantes infinitas de un tema único: cambiar la faz de la América, y sobre todo, de la República Argentina, por la sustitución del espíritu europeo a la tradición española; y a la fuerza bruta como móvil, la inteligencia cultivada, el estudio y el remedio de las necesidades (pp. 215-216).

El hijo, el hermano y el amigo. Progreso, Santiago; 10 pages". En el t. 1 de Obras, Santiago de Chile, Gutenberg, 1887, en la "Bibliografía de las publicaciones que hizo en Chile el señor Sarmiento" reunida por Luis Montt, pp. xvn-xvm se cita para el año 1843, en el núm. 14 "Vaya un refresco..." $\mathrm{y}$ en el núm. 15, Mi defensa, donde después de consignar las partes 1, 2 y 3 ya tradicionales, agrega: "4. El libelo. No conocíamos la 4a Parte cuando reprodujimos la Defensa al principio del tomo 3 de las Obras" (recuérdese que el t. 3 fue el primero de la colección en ser publicado, en 1885). Según comentario de Raúl Mogria y Miguel García en Sarmiento. Educador. Sociólogo. Escritor. Político, Buenos Aires, 1963, p. 200, "Vaya un refresco..." se introdujo en la reimpresión de las Obras, t. 3 de 1903 y "El escritor en Chile" en la de 1913. No he podido verificar personalmente esta información. 
Educación personal y educación de todos, lucha por la propia vida y por cambiar la vida de la patria (y aun de América). Recuerdos de provincia acaba con otro enfrentamiento Rosas-Sarmiento, que esta vez se da bajo la metáfora vida-libro y su inversa libro-vida. Se ve la propia vida como la continuada escritura de una serie de textos, que son también actos y construyen la historia, proyectándola hacia el futuro. Con calculada estrategia el autor presenta el futuro de su libro-vida, vida-libro dramáticamente amenazado por Rosas.

[Recuerdos] Este opúsculo, pues, es el prólogo de una obra apenas comenzada. Llámase el primer volumen Viajes por Europa, Africa y América. El segundo está todavía en manos de la Providencia. Don Juan Manuel de Rosas pretende que no ha de publicarse sin su visto bueno, y que él sabe desparpajar los libros en su fuente. ¡Florencio Varela! ¿estáis también en el secreto? (p. 216).

No es la única vez que Sarmiento se identifica como hombre con su obra escrita y con el destino de su tierra. En el "Epílogo" de la Campaña en el Ejército Grande (316) vuelve a poner la serie de sus libros y periódicos, Civilización y barbarie, Crónica, Argirópolis, Sud América, y también la Campaña "que son sólo capítulos de un mismo libro" (porque hay una rotunda unidad en su vida y en su obra conjugadas) y los ve sucumbir cubiertos por el polvo y arrastrados junto con él en la ecatombe de su patria, si triunfa el "caudillaje" con Urquiza. Así, señoreándola; se instala Sarmiento en la escena de la escritura, y se prepara para ejercer su dominio en la escena de la acción política.

Ana María Barrenechea

Columbia University. 